\title{
Vergleich zweier Verfahren der Unsicherheitsabschätzung bei linearisierten inversen Problemen mittels Bayes'scher Inferenz und Pseudoinverse am Beispiel akustischer Transmissionsmessung \\ Nadine Feldmann ${ }^{1}$, Fabian Bause ${ }^{1}$, Bernd Henning ${ }^{1}$ \\ ${ }^{1}$ Elektrische Messtechnik, Universität Paderborn, Warburger Straße 100, Paderborn feldmann@emt.upb.de, bause@emt.upb.de, henning@emt.upb.de
}

\begin{abstract}
Zusammenfassung
Die simulative Entwicklung akustischer Sensorsysteme erfordert unumgänglich die Kenntnis des Verhaltens zugrunde liegender Materialien. Die Bestimmung dieser akustischen Materialparameter durch geführte Wellen kann als ein inverses Messverfahren realisiert werden. Die resultierenden Materialparameter, welche simulativ das gemessene Signal am besten abbilden, werden dann zur Materialbeschreibung herangezogen. Um eine Unsicherheit dieser gefundenen Lösung anzugeben, soll hier ein stochastischer Ansatz verfolgt werden. Zunächst ist dazu ein Übergang von einer deterministisch-simulativen zu einer statistischen Beschreibung des Messsystems notwendig. Dabei sollen hier zwei unterschiedliche Verfahren betrachtet werden - eine Unsicherheitsabschätzung mittels Bayes'scher Inferenz und eine mittels Pseudoinverse. Dabei wird die Annahme eines linearisierten Messsystems und ausschließlich gaußverteilter Unsicherheiten vertreten. Die sich stark unterscheidenden Ergebnisse beider Verfahren werden am Anwendungsbeispiel diskutiert und interpretiert.
\end{abstract}

Keywords: Statistische inverses Verfahren, Bayes'sche Inferenz, Pseudoinverse, geführte akustische Wellen, Materialparameterbestimmung

\section{Einleitung}

Beim Design akustischer Sensor- und Messsysteme gewinnen simulative Untersuchungen in den letzten Jahren stetig an Bedeutung. Die Kenntnis akustischer Materialdaten ist bei der Modellierung des Systems unumgänglich. Ihre Bestimmung ist jedoch hauptsächlich für quasistatische Messverfahren standardisiert (DIN EN ISO 899, DIN EN ISO 6721) und bildet das Materialverhalten daher auch nur in diesem Frequenzbereich hinreichend gut ab. Eine Extrapolation der quasi-statischen Messwerte auf akustisch relevante Frequenzen ist dabei nur bedingt möglich. Eine Bestimmung akustischer Materialparameter ist somit auch über ein akustisches Messsystem sinnvoll. Das hier beschriebene Verfahren fußt auf akustische Transmissionsmessungen durch einen Hohlzylinder [1][2]. Die gemessenen Signale werden dann mit einer Simulation verglichen, deren Materialparameter optimiert werden, sodass eine möglichst gute Übereinstimmung zwischen Messsignal und Simulationssignal erreicht wird. Dieses Vorgehen lässt sich als inverses Problem beschreiben. Die Simulation stellt das sogenanntes Vorwärtsmodell dar, welches das Eingangssignal $\boldsymbol{x}$ in Abhängigkeit der Materialparametern $\boldsymbol{\theta}$ auf das Ausgangssignal $\boldsymbol{y}$ abbildet:

$$
y=f(x, \theta)
$$

Somit ist für jedes $\boldsymbol{\theta}$ ein Ausgangssignal gegeben. Die Lösung des inversen Problems ist nun genau anders herum eine Materialparametersatz $\boldsymbol{\theta}$ zu einem gegebenen Ausgangssignal $\boldsymbol{y}$ [3]. Diese Lösung ist jedoch nicht immer eindeutig definiert. Außerdem ist das reale Messsignal $\boldsymbol{y}_{\text {mess }}$ stets unsicherheitsbehaftet, sodass das gegebene Vorwärtsmodell das reale Messsystem nur im Rahmen dieser Unsicherheit beschreiben kann. Die Lösung des inversen Problems ist daher dasjenige $\boldsymbol{\theta}$, welches die quadratische Norm der Differenz zwischen gemessenem und simuliertem Signal minimiert. Um die Eindeutigkeit der Lösung zu gewährleisten, müssen gegebenenfalls weitere Informationen zur Verfügung gestellt werden. Dies wird als Regularisierung des inversen Problems bezeichnet. Die Frage nach der Unsicherheit der ermittelten Lösung, welche aus den Unsicherheiten des Messsignals resultieren, kann durch eine statistische Beschreibung des zugrundeliegenden Systems beantwortet werden.

\section{Statistische Beschreibung inverser Prob- leme durch Bayes'sche Inferenz (BI)}

Der Übergang von einer deterministischen zu einer statistischen Modellbeschreibung ge- 
schieht zunächst wieder über das Vorwärtsmodell. Für ein gegebenes $\boldsymbol{\theta}$ wird nun als Äquivalent für das deterministische Ausgangssignal $\boldsymbol{y}$ die Wahrscheinlichkeit für ein auftretendes unsicherheitsbehaftetes Ausgangssignal $\boldsymbol{y}_{\text {mess }}$ über die Likelihood $p\left(\boldsymbol{y}_{\text {mess }} \mid \boldsymbol{\theta}\right)$ angegeben. Aus der Kenntnis des deterministischen Vorwärtsmodells und der statistischen Beschreibung der auftretenden Unsicherheiten ist diese stets definiert. Das Ergebnis der Inversion ist dann gegeben durch die A-PosterioriWahrscheinlichkeitsdichte $p\left(\boldsymbol{\theta} \mid \boldsymbol{y}_{\text {mess }}\right)$, welche analog bei einem gegebenen Messsignal die Wahrscheinlichkeit für zugrundeliegende Materialparameter $\boldsymbol{\theta}$ angibt. Diese lässt sich aus der gegebenen Likelihood mit Hilfe der Bayes'schen Regel berechnen [4].

$p(\boldsymbol{\theta} \mid \boldsymbol{y}) \propto p(\boldsymbol{y} \mid \boldsymbol{\theta}) \cdot p(\boldsymbol{\theta})$

$p(\boldsymbol{\theta})$ bezeichnet hier die A-Posterior-Wahrscheinlichkeitsdichte, welche das Vorwissen abbildet, d.h. Eigenschaften der Materialparameter, welche bekannt sind bevor Messungen durchgeführt wurden. Die gesuchten deterministischen Größen werden im statistischen Äquivalent folglich durch Wahrscheinlichkeitsdichten ersetzt. Zunächst ist die gesamte APosteriori-Wahrscheinlichkeitsdichte als Lösung des statistischen inversen Problems anzusehen. Da diese jedoch beliebige Form und Dimension annehmen kann, ist diese zur Angabe von Unsicherheiten meist nicht praktikabel, sodass zunächst ein Unsicherheitsmaß im Sinne der Standardabweichungen der Elemente von $\boldsymbol{\theta}$ aus dieser berechnet werden soll.

\section{Bayes'sche Inferenz bei linearisierten in- versen Problemen und gaußverteilten Unsi- cherheiten}

Die Berechnung der Standardabweichung kann schnell zu numerischen Problemen und sehr langen Rechenzeiten führen. Um dies zu umgehen, sollen hier folgende Annahmen getroffen werden:

- Alle Wahrscheinlichkeitsdichten können als Gaußverteilungen dargestellt werden.

- Das Vorwärtsmodell kann in einem Arbeitspunkt linearisiert werden und ist somit durch

$\boldsymbol{y}=\boldsymbol{A \theta}+\boldsymbol{b}$

gegeben.

Die Tatsache, dass alle Wahrscheinlichkeitsdichten als gaußverteilt angenommen werde, lässt sich mathematisch beschreiben durch

$$
p(\boldsymbol{\theta})=\mathcal{N}\left(\boldsymbol{\theta} ; \boldsymbol{\mu}_{0}, \boldsymbol{\Sigma}_{0}\right)
$$

$p\left(\boldsymbol{y}_{\text {mess }} \mid \boldsymbol{\theta}\right)=\mathcal{N}\left(\boldsymbol{y}_{\text {mess }} ; \boldsymbol{A} \boldsymbol{\theta}+\boldsymbol{b}, \boldsymbol{\Sigma}_{\mathrm{u}}\right)$

, wobei $\mathcal{N}(\boldsymbol{x} ; \boldsymbol{\mu}, \boldsymbol{\Sigma})$ angibt, dass $\boldsymbol{x}$ normalverteilt ist mit Mittelwertvektor $\boldsymbol{\mu}$ und Kovarianzmatrix $\boldsymbol{\Sigma} . \boldsymbol{\Sigma}_{\mathrm{u}}$ fasst alle auftretenden Unsicherheiten des Messsystems zusammen. Für diesen speziellen Fall lässt sich die gesuchte A-PosterioriWahrscheinlichkeitsdichte analytisch wieder zu einer Gaußverteilung bestimmen [4]:

$p\left(\boldsymbol{\theta} \mid \boldsymbol{y}_{\text {mess }}\right)=\mathcal{N}\left(\boldsymbol{\theta} ; \boldsymbol{\mu}^{\mathrm{BI}}, \boldsymbol{\Sigma}^{\mathrm{BI}}\right)$

, mit

$\boldsymbol{\Sigma}^{\mathrm{BI}}=\left(\boldsymbol{A}^{\mathrm{T}} \boldsymbol{\Sigma}_{\mathrm{u}}^{-1} \boldsymbol{A}+\boldsymbol{\Sigma}_{0}^{-1}\right)^{-1}$

$\boldsymbol{\mu}^{B I}=\boldsymbol{\Sigma}^{\mathrm{BI}}\left(\boldsymbol{A}^{\mathrm{T}} \boldsymbol{\Sigma}_{\mathrm{u}}^{-1}\left(\boldsymbol{y}_{\text {mess }}-\boldsymbol{b}\right)+\boldsymbol{\Sigma}_{0}^{-1} \boldsymbol{\mu}_{0}\right)$.

Somit lässt sich unter den gegebenen Annahmen die A-Posteriori-Wahrscheinlichkeitsdichte und auch ihre Standardabweichung bzw. Varianz stets durch einfache Rechenoperationen angeben.

\section{Statistische Beschreibung inverser Prob- leme mittels Pseudoinverse (PI)}

Eine Alternative zur Bayes'schen Inferenz bietet ein Ansatz mit Hilfe der Moore-PenrosePseudoinversen [5]. Auch hier wird wieder von einem linearen System und der Vergleichbarkeit halber von gaußverteilten Wahrscheinlichkeitsdichten ausgegangen. Die kleinste Fehlerquadratlösung des inversen Problems, welche im deterministischen Sinne die Lösung des inversen Problems beschreibt, lässt sich für lineare Systeme analytisch angeben:

$\boldsymbol{\theta}^{\mathrm{PI}}=\boldsymbol{A}^{+}\left(\boldsymbol{y}_{\text {mess }}-\boldsymbol{b}\right)$

, wobei $\boldsymbol{A}^{+}$die Pseudoinverse zur Matrix $\boldsymbol{A}$ ist. Ist das Gleichungssystem, welches durch die Matrix $\boldsymbol{A}$ repräsentiert wird unterbestimmt, existiert mehr als eine Lösung. Mit Hilfe der Pseudoinverse erhält man dann stets die Lösung, welche die Norm von $\boldsymbol{\theta}$ minimiert. Unter anderem lässt sich die Pseudoinverse effizient über eine Singulärwertzerlegung berechnen. Somit beschreibt Gl. (9) nun eindeutig ein Vorwärtsmodell, welches direkt die gewünschten Parameter $\boldsymbol{\theta}$ liefert. Die Beschreibung von Unsicherheiten im Vorwärtsmodell folgt den Richtlinien des "Guide to the expression of uncertainty in measurement" (GUM) [6][7]. Die Unsicherheiten des Messsignals werden durch $\boldsymbol{\Sigma}_{\mathrm{u}}$ modelliert und können somit durch Gl. (11) 
auf die Unsicherheit von $\boldsymbol{\theta}$ bei gegebenem $\boldsymbol{y}_{\text {mess }}$ abgebildet werden:

$\boldsymbol{\mu}^{\mathrm{PI}}=\boldsymbol{A}^{+}\left(\boldsymbol{y}_{\text {mess }}-\boldsymbol{b}\right)$

$\Sigma^{\mathrm{PI}}=A^{+} \Sigma_{\mathrm{u}} A^{+, \mathrm{T}}$

Modellierung von Unsicherheiten am Beispiel akustischer Transmissionsmessung

Hier soll das Beispiel einer akustischen Transmissionsmessung durch einen polymeren Hohlzylinder zur Materialdatencharakterisierung betrachtet werden (siehe Abb. 1). Dazu wird an den Stirnflächen eines Probekörper aus Polypropylen eine akustische Welle mittels eines piezoelektrischen Schallwandlers eingekoppelt. Als Sendesignal $\boldsymbol{x}_{0}$ wurde dabei ein breitbandiger Puls mit einer Mittenfrequenz von $1 \mathrm{MHz}$ gewählt, um eine Vielzahl an Moden anzuregen. An der gegenüberliegenden Seite dient ein zweiter Wandler dazu, das Messsignal $\boldsymbol{y}_{\text {mess }}$ integral über die Fläche aufzunehmen. Der gesamte Messprozess kann als unsicherheitsbehaftet angesehen werden.

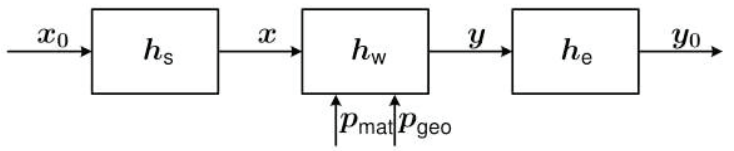

Abb. 1: Messstrecke

Als Vorwärtsmodell dient in diesem Fall eine semi-analytische Simulation [1]. Es wird davon ausgegangen, dass das dynamische Verhalten der Schallwandler, welche durch $\boldsymbol{h}_{\mathrm{s}}$ und $\boldsymbol{h}_{\mathrm{e}}$ dargestellt sind, bekannt ist und ihr Einfluss eliminiert werden kann. Somit ist im Folgenden nur das System $\boldsymbol{h}_{\mathrm{w}}$ von Interesse. Dieses hängt unter anderen von einem bekannten Eingangssignal, den gesuchten Materialeigenschaften des Probekörpers $\boldsymbol{p}_{\text {mat }}$ und den Geometrieabmessungen des Probekörpers $\boldsymbol{p}_{\text {geo }}$ ab. Die Materialeigenschafen sind in diesem Fall die $\mathrm{zu}$ bestimmenden Parameter $\boldsymbol{\theta}=\boldsymbol{p}_{\text {mat }}$. Durch einen Optimierungsalgorithmus sei hier schon ein bester Schätzwert $\widehat{\boldsymbol{p}}_{\text {mat }}^{\text {opt }}$ für diese gefunden, sodass das System in diesem Punkt linearisiert werden kann.

Die auftretenden Unsicherheiten seien hier auf drei Unsicherheitsquellen beschränkt. Zum einen wird additives, weißes, gaußverteiltes Messrauschen $\boldsymbol{r}=\mathcal{N}\left(\boldsymbol{r} ; 0, \sigma_{r}^{2} \boldsymbol{I}\right)$ angenommen, wobei $I$ die Einheitsmatrix beschreibt und $\sigma_{r}^{2}=$ $1,556 \cdot 10^{-5}$ durch Mehrfachmessung des normierten Messsignals bestimmt werden konnte. Zum anderen gilt es, die Unsicherheiten der Geometrieabmessungen des Wellenleiters und der Dicke der Koppelschicht, welche für eine bessere Einkopplung der Schallwelle zwischen
Schallwandler und Probekörper aufgebracht wird, zu berücksichtigen. Beschrieben werden soll dieses unsicherheitsbehaftete System durch

$\boldsymbol{y}_{\mathrm{mess}}=\boldsymbol{y}+\boldsymbol{r}+\boldsymbol{r}_{\mathrm{geo}}+\boldsymbol{r}_{\mathrm{coup}}$.

Das Messrauschen ist bereits definiert. Zunächst sollen nun eine statistische Beschreibung der Geometrieabmessungen bestehend aus Innenradius $r_{\mathrm{i}}$, Außenradius $r_{\mathrm{a}}$ und Länge $l$ des Wellenleiters vorgenommen werden. Diese drei Größen werden der Einfachheit halber als unkorreliert betrachtet, sodass ihre Kovarianzmatrix Diagonalform annimmt:

$\boldsymbol{\Sigma}_{\text {geo }}=\operatorname{diag}\left(\sigma_{r_{\mathrm{i}}}^{2}, \sigma_{r_{\mathrm{a}}}^{2}, \sigma_{l}^{2}\right)$

Nun lässt sich das Vorwärtsmodell in den drei Geometrieparametern linearisieren, sodass es durch

$\boldsymbol{y}=\boldsymbol{A}_{\mathrm{geo}} \boldsymbol{p}_{\mathrm{geo}}+\boldsymbol{b}_{\mathrm{geo}}$

abgebildet wird. Damit lässt sich nun die Unsicherheit der Geometriegrößen auf eine Unsicherheit des Messsignals abbilden [JCGM11]:

$\Sigma_{r, \text { geo }}=A_{\text {geo }} \Sigma_{\text {geo }} A_{\text {geo }}^{\mathrm{T}}$

Somit sind auch hier die Unsicherheiten dargelegt. Als letzte Unsicherheitsquelle muss nun noch die Kovarianzmatrix $\boldsymbol{\Sigma}_{r \text {,coup }}$ bestimmt werden. Dies geschieht analog zu den Geometrieparametern durch Propagation der Unsicherheiten, wie sie in der GUM beschrieben wird. Das Verhalten, welches sich durch eine veränderte Koppelschicht ergibt, kann durch eine Verschiebung des Messsignals erreicht werden. Dabei konnte das 95\%-Konfidenzintervall der Verschiebezeit mit $t_{\text {coup }}=$ $\left[-\frac{1}{f_{\mathrm{s}}} ; \frac{1}{f_{\mathrm{s}}}\right]$ identifiziert werden. Dies entspricht für eine Gaußverteilung einer Standardabweichung von $\sigma_{\text {coup }}=\frac{1}{1,96 f_{\mathrm{s}}}$ mit einer Abtastfrequenz von $f_{\mathrm{s}}=50 \mathrm{MHz}$ [6]. Wird dies durch das linearisierte System propagiert, erhält man $\boldsymbol{\Sigma}_{r \text {,coup }}$. Damit sind nun alle benötigten Größen modelliert. All diese Unsicherheiten lassen sich nun noch zusammenfassen, sodass das Messsignal über

$\boldsymbol{y}_{\text {mess }}=\boldsymbol{y}+\boldsymbol{r}_{\text {ges }}$
$\boldsymbol{r}_{\text {ges }}=\mathcal{N}\left(\boldsymbol{r}_{\text {ges }} ; 0, \boldsymbol{\Sigma}_{r}+\boldsymbol{\Sigma}_{r, \text { geo }}+\boldsymbol{\Sigma}_{r, \text { coup }}\right)$

beschrieben wird. Ein sich so ergebendes Signal ist in Abb. 2 dargestellt. 


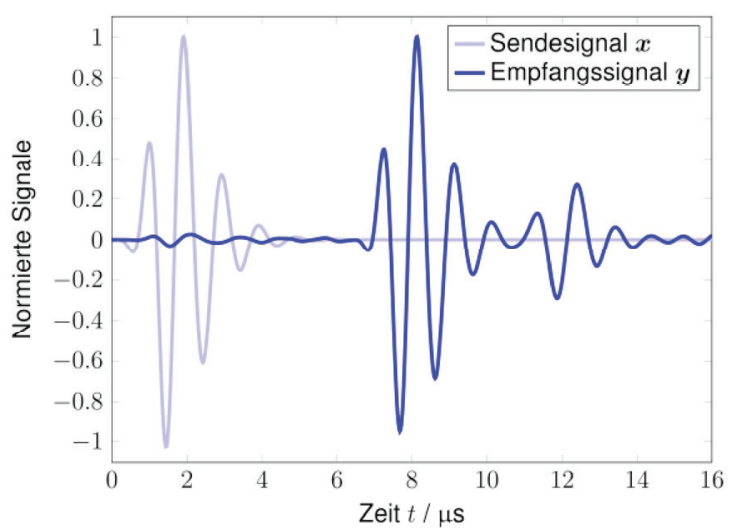

Abb. 2: Beispielhaftes Sende- und Empfangssignal

Abb. 3 zeigt die Einflüsse der einzelnen Unsicherheitsquellen auf das Ausgangssignal y. Dabei fällt auf, dass die Dicke der Koppelschicht die größte Unsicherheitskomponente darstellt.

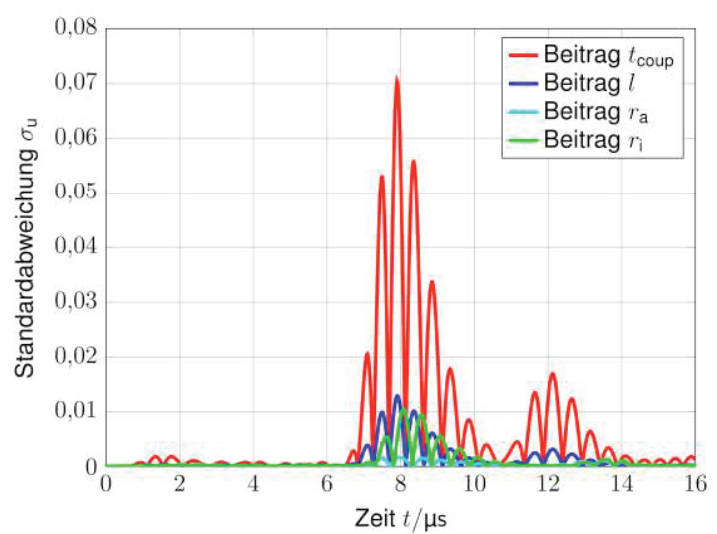

Abb. 3: Unsicherheitsbeiträge der Geometrieund Koppelschichtgrößen

Ergebnisse zur Schätzung der Schallgeschwindigkeit und der Poissonzahl

Für einen konkreten Messaufbau wurde eine isotrope Polypropylenprobe betrachtet, für die die Schallgeschwindigkeit $c$ und die Poissonzahl $v$ über das oben beschriebene Messsystem bestimmt werden soll. Eine Optimierung liefert vorab einen Arbeitspunkt $\left(c^{\mathrm{opt}}, v^{\mathrm{opt}}\right)$ um den linearisiert wurde. Dieser sowie die geometrischen Abmessungen sind in Tab. 1 angegeben. Das notwendige Vorwissen wurde hier so gewählt, dass sein Einfluss auf die Ergebnisse vernachlässigbar ist. Dies geschieht durch eine in Relation zur Likelihood sehr breit gewählte A-Priori-Wahrscheinlichkeitsdichte. Um zunächst eine Erwartungshaltung zu entwickeln, kann durch einfache physikalische Überlegungen abgeschätzt werden, wie groß die Unsicherheit beispielsweise in der Schallgeschwindigkeit anzunehmen ist.
Tab. 1: Geometrieabmessungen und Arbeitspunkt der Materialkenngrößen

\begin{tabular}{|c|c|c|}
\hline Größe & Mittelwert & Streuung \\
\hline$r_{i}$ & $3 \mathrm{~mm}$ & $2,291 \mu \mathrm{m}$ \\
\hline$r_{a}$ & $9 \mathrm{~mm}$ & $2,386 \mu \mathrm{m}$ \\
\hline$l$ & $16,9 \mathrm{~mm}$ & $5,058 \mu \mathrm{m}$ \\
\hline$c^{\text {opt }}$ & $2690 \mathrm{~m} / \mathrm{s}$ & - \\
\hline$v^{\text {opt }}$ & 0.342 & - \\
\hline
\end{tabular}

Zunächst soll ausschließlich die Koppelschichtdicke als Unsicherheitsbeitrag angenommen werden, da diese wie in Abb. 3 den größten Unsicherheitsbeitrag darstellt. Da diese als Verschiebungszeit von 20 ns modelliert wurde und die Länge des Wellenleiters bekannt ist, kann über den einfachen Zusammenhang $c=l / t$ eine grobe Abschätzung der Unsicherheit der Schallgeschwindigkeit zu $4 \mathrm{~m} / \mathrm{s}$ angeben werden. Für die Poissonzahl ergibt eine ähnliche Abschätzung eine Größenordnung von ca. $10^{-4}$. Die Ergebnisse, welche sich durch Bayes'sche Inferenz und Pseudoinversenansatz ergeben, sind in Tab. 2 zusammengefasst.

Tab. 2: Vergleich der Ergebnisse der Berechnungen mit unterschiedlichen Verfahren: Mittelwerte $\mu$ und Standardabweichungen $\sigma$ der Schallgeschwindigkeit $c$ und der Poissonzahl $v$

\begin{tabular}{|c|c|c|c|c|}
\hline & $\mu_{c} / \mathrm{m} / \mathrm{s}$ & $\sigma_{c} / \mathrm{m} / \mathrm{s}$ & $\mu_{v}$ & $\sigma_{v}$ \\
\hline $\mathrm{BI}$ & 2690,03 & 0,041 & 0,3419 & $2,8 \cdot 10^{-5}$ \\
\hline $\mathrm{PI}$ & 2693,73 & 4,299 & 0,3421 & $3,7 \cdot 10^{-4}$ \\
\hline
\end{tabular}

Man erkennt, dass die gefundenen Ergebnisse stark variieren. Außerdem scheint der Ansatz mittels Pseudoinverse eher den Erwartungen zu entsprechen. Um diesen Unterschied zu interpretieren soll zunächst ein genereller Vergleich beider Verfahren durchgeführt werden.

\section{Vergleich beider Verfahren zur Unsicher- heitsabschätzung}

Der offensichtlichste Unterschied beider Verfahren ist, dass die Bayes'sche Inferenz das Einbringen von Vorwissen fordert. Um einen Vergleich mit der Pseudoinverse zu ermöglichen, kann dieser Einfluss mathematisch durch $\Sigma_{0}^{-1}=0$ ausgeblendet werden.

Um die Berechnungen der Schätzwerte, welche in Tab. 3 zusammengefasst sind, anschaulicher erörtern zu können, lassen sich beide als Optimierung unterschiedlicher Kostenfunktionen interpretieren. 
Tab. 3: Berechnung der Schätzwerte Mittelwert und Kovarianzmatrix

\begin{tabular}{|c|c|c|}
\hline & $\boldsymbol{\mu}$ & $\boldsymbol{\Sigma}$ \\
\hline $\mathrm{BI}$ & $\boldsymbol{\Sigma}^{\mathrm{BI}}\left(\boldsymbol{A}^{\mathrm{T}} \boldsymbol{\Sigma}_{\mathbf{u}}^{-1}\left(\boldsymbol{y}_{\text {mess }}-\boldsymbol{b}\right)\right.$ & $\left(\boldsymbol{A}^{\mathrm{T}} \boldsymbol{\Sigma}_{\mathrm{u}}^{-1} \boldsymbol{A}\right.$ \\
& $\left.+\boldsymbol{\Sigma}_{0}^{-1} \boldsymbol{\mu}_{0}\right)$ & $\left.+\boldsymbol{\Sigma}_{0}^{-1}\right)^{-1}$ \\
\hline $\mathrm{PI}$ & $\boldsymbol{A}^{+}\left(\boldsymbol{y}_{\text {mess }}-\boldsymbol{b}\right)$ & $\boldsymbol{A}^{+} \boldsymbol{\Sigma}_{\mathrm{u}} \boldsymbol{A}^{+, \mathrm{T}}$ \\
\hline
\end{tabular}

Die Kostenfunktion, welche durch $\boldsymbol{\mu}^{\mathrm{BI}}$ minimiert wird (bei Vernachlässigung von Vorwissen) ist:

$$
\begin{gathered}
\boldsymbol{\mu}^{\mathrm{BI}}=\underset{\boldsymbol{\theta}}{\operatorname{argmin}}\left(\boldsymbol{y}_{\text {mess }}-\boldsymbol{A} \boldsymbol{\theta}-\boldsymbol{b}\right)^{\mathrm{T}} \cdot \boldsymbol{\Sigma}_{\mathbf{u}}^{-1} . \\
\left(\boldsymbol{y}_{\text {mess }}-\boldsymbol{A} \boldsymbol{\theta}-\boldsymbol{b}\right)
\end{gathered}
$$

Im Vergleich dazu stellt der Pseudoinversenansatz das Minimum folgender Kostenfunktion dar:

$$
\begin{array}{r}
\boldsymbol{\mu}^{\mathrm{PI}}=\underset{\boldsymbol{\theta}}{\operatorname{argmin}}\left(\boldsymbol{y}_{\text {mess }}-\boldsymbol{A} \boldsymbol{\theta}-\boldsymbol{b}\right)^{\mathrm{T}} \cdot \boldsymbol{I} . \\
\left(\boldsymbol{y}_{\text {mess }}-\boldsymbol{A} \boldsymbol{\theta}-\boldsymbol{b}\right)
\end{array}
$$

Offensichtlich wird bei der Bayes'schen Inferenz die Abweichung zwischen Messung und Vorwärtsmodell $\left(\boldsymbol{y}_{\text {mess }}-\boldsymbol{A} \boldsymbol{\theta}-\boldsymbol{b}\right)$ mit Hilfe der vorhandenen Unsicherheiten gewichtet. Das bedeutet also, dass Zeitabschnitte des Ausgangssignals, welche mit geringeren Unsicherheiten behaftet sind, stärker in die Schätzung mit einbezogen werden, also solche, die große Unsicherheiten aufweisen. Im Allgemeinen bedeutet dies, dass bei der Bestimmung der APosteriori-Wahrscheinlichkeitsdichte mehr Informationen zur Verfügung stehen als beim Pseudoinversenansatz. Diese zusätzlichen Informationen sollten generell dafür sorgen, dass die Unsicherheitsabschätzung bessere Ergebnisse liefert. In dem hier gewählten Beispiel ist jedoch das Gegenteil der Fall, sodass sich für die durch Bayes'sche Inferenz ermittelten Unsicherheiten unplausible Werte ergeben. Der Grund dafür liegt hier in der zweimaligen Propagation der Unsicherheiten. Zunächst wird im Sinne der GUM [6] die Unsicherheit der Geometrie- und Koppelschichtgrößen auf eine Unsicherheit des Ausgangssignals abgebildet. Dies geschieht mittels Linearisierung, welche gerechtfertigt ist, da diese Approximation im Bereich der Unsicherheit der Geometriegrößen das nicht-lineare Simulationsmodell gut abbildet. Durch die Linearisierung entsteht eine gewisse Skalierung des Ausgangssignals bei Änderung der Geometrieabmessungen, welche jedoch so gering ist, dass die Unsicherheiten des Ausgangssignals hinreichend gut abschätzbar sind. Es kann also davon ausgegangen werden, dass die sich ergebene Kovarianzmatrix $\boldsymbol{\Sigma}_{\mathrm{u}}$ sowohl die Unsicherheiten des linearen, als auch des nicht-linearen Systems gut beschreibt. Wird nun die statistische Inversion vorgenommen. An diesem Punkt wird bei der Bayes'schen Inferenz die Kovarianzmatrix $\boldsymbol{\Sigma}_{\mathrm{u}}$ jedoch nicht wie beim Ansatz mittels Pseudoinverse nur für die Bestimmung der Unsicherheiten der Materialparameter, sondern auch für die Bestimmung eines Mittelwertes genutzt. In Tab. 3 wird dies in der Spalte für die Berechnung des Mittelwertes $\boldsymbol{\mu}$ deutlich, da hier bei der Pseudoinverse kein Wissen über die Unsicherheiten notwendig ist. In diesem Fall geschieht die Schätzung ausschließlich auf Basis des gemessenen Signals $\boldsymbol{y}_{\text {mess }}$ und der Systembeschreibung durch $\boldsymbol{A}$ und $\boldsymbol{b}$. Im Gegensatz dazu benötigt die Bayes'sche Inferenz auch die Unsicherheitsbeschreibung $\boldsymbol{\Sigma}_{\mathrm{u}}$. Die Approximation ist zwar hinreichend genau, um die Unsicherheiten des Ausgangssignals $\boldsymbol{y}_{\text {mess }}$ anzugeben, wird dies allerdings nun für die Inversion genutzt, sorgt in diesem Fall die vorher vernachlässigbare Skalierung dafür, dass gerade diese bei der Berechnung des Mittelwertes jegliche Geometrie- und Koppelschichtunsicherheiten eliminieren kann. Die zur Beschreibung der Unsicherheiten hinreichende Approximation ist für die Inversion dementsprechend nicht mehr sinnvoll, da Sie dabei zusätzlich den Mittelwert beeinflusst. Diese Beeinflussung des Mittelwerts ist gerade der Unterschied zur Berechnung mittels Pseudoinverse. Hier wird die durch lineare Approximation bestimmte Unsicherheit des Ausgangssignals $\boldsymbol{\Sigma}_{\mathrm{u}}$ weiterhin nur als Maß für die Unsicherheit, jedoch nicht zur Bestimmung des Mittelwerts selbst genutzt, sodass die Approximation weiterhin hinreichend ist. Daher ergeben sich im Beispiel der akustischen Transmissionsmessung mit Hilfe der Pseudoinverse plausiblere Ergebnisse.

Beide Verfahren liefern jedoch für zwei Sonderfälle identische Ergebnisse. Zum einen ist dies der Fall, wenn $\boldsymbol{\Sigma}_{\mathrm{u}}$ eine skalierte Einheitsmatrix ist, d.h. $\boldsymbol{\Sigma}_{\mathrm{u}}=\sigma_{\mathrm{u}}^{2} \boldsymbol{I}$. Der Schätzwert $\boldsymbol{\mu}$ ist hier identisch, da die zu minimierenden Kostenfunktionen (GI. (18) und Gl. (19)) hier auch nur skalierte Varianten voneinander sind. Eine Skalierung beeinflusst jedoch nicht die Lage des Minimums. Zum anderen sind beide Verfahren identisch, wenn die Matrix $\boldsymbol{A}$ invertierbar ist. Wenn dies der Fall ist, ist das inverse Problem gut-gestellt, d.h. es kann für jedes gegebene $\boldsymbol{y}$ ein eindeutiges $\boldsymbol{\theta}$ gefunden werden. Eine Inversion ist dann problemlos analytisch möglich. Die Pseudoinverse geht in die Inverse über. 


\section{Zusammenfassung}

Zusammenfassend kann festgestellt werden, dass sich im hier betrachteten Fall erhebliche Unterschiede bei den resultierenden Unsicherheiten für die Materialparameter mit unterschiedlichen Verfahren ergeben. Der prinzipielle Unterschied wird deutlich, wenn man die Schätzer, welche die beiden Verfahren charakterisieren, als Minimierung einer Kostenfunktion interpretiert. Dann ergibt der Mittelwert jeweils das Minimum der Norm der Differenz zwischen Mess- und Simulationssignal, wobei die Bayes'sche Inferenz diese noch mit den vorhandenen Unsicherheiten gewichtet. Diese Gewichtung sollte zunächst als positiv gewertet werden, da so mehr Informationen zur Verfügung stehen. Bei der beispielhaft angeführten Transmissionsmessung führt diese jedoch zu unplausiblen Ergebnissen, da $\boldsymbol{\Sigma}_{\mathrm{u}}$ mit Hilfe eines linearisierten Systems bestimmt wurde. Die vorgenommene Approximation ist hinreichend genau, um eine Unsicherheitsabschätzung zu ermöglichen, allerdings nicht um diese bei der Inversion mit in die Schätzung einzubinden. Der Ansatz mittels Pseudoinverse verfolgt genau diesen Ansatz und nutzt $\boldsymbol{\Sigma}_{\mathrm{u}}$ nur zur Abbildung der Messunsicherheiten auf die Unsicherheiten der Materialparameter, aber nicht zur Gewichtung bzw. zur Bestimmung des Schätzwertes. Weiterhin lässt sich dieser Ansatz leicht im Rahmen der GUM [6] interpretieren, da zunächst die Inversion vorgenommen wird und somit das invertierte System analog zu einem Vorwärtsmodell beschrieben wird.

\section{Danksagung}

Die Autoren bedanken sich bei der Deutschen Forschungsgemeinschaft für die finanzielle Unterstützung des Forschungsprojekts HE 2897/31 (Bestimmung komplexer akustischer Materialkenngrößen) und HE 2897/6-1 (Ermittlung des hydrothermischen Alterungsverhaltens endlosfaserverstärkter Thermoplaste und Entwicklung eines ultraschallbasierten Messsystems zur zerstörungsfreien Charakterisierung des Alterungszustands für die Komponentenüberwachung und Restlebenszeitprädiktion).

\section{Literaturnachweis}

[1] Rautenberg, J.: Ein wellenleiterbasiertes Verfahren zur Bestimmung von Materialdaten für die realitätsnahe Simulation von Schallausbreitungsphänomenen am Beispiel stark absorbierender Kunststoffe, Universität Paderborn Dissertation. Paderborn 2012

[2] Bause, F., Weber, D., Rautenberg, J. u. Henning, B.: Unsicherheitsanalyse eines Vorwärtsmodells zur Simulation transienter Wellenausbreitung im Hohlzylinder. Sensoren und Messsysteme 2014. Beiträge der 17.
ITG/GMA-Fachtagung vom 3. bis 4. Juni 2014 in Nürnberg. ITG-Fachbericht, Bd. 250. Berlin, Offenbach: VDE-Verl. 2014

[3] Louis, A. K.: Inverse und schlecht gestellte Probleme. Teubner-Studienbücher : Mathematik. Stuttgart: Teubner 1989; doi: 10.1007/9783-322-84808-6

[4] Kaipio, J. u. Somersalo, E.: Statistical and Computational Inverse Problems. Applied Mathematical Sciences, Bd. 550. New York, NY: Springer New York 2004; doi: 10.1007/b138659

[5] Penrose, R. u. Todd, J. A.: A generalized inverse for matrices. Mathematical Proceedings of the Cambridge Philosophical Society 51 (1955) 03, S. 406-413; doi: $10.1017 /$ S0305004100030401

[6] JCGM 100:2008 100:2008; 2008. Evaluation of measurement data - Guide to the expression of uncertainty in measurement

[7] JCGM 102:2011; 2011. Evaluation of measurement data - Supplement 2 to the "Guide to the expression of uncertainty measurement"- Extension to any number of output quantities. 